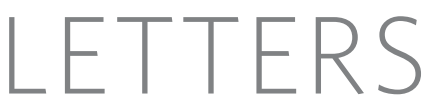

\title{
Genome-wide RNAi screen identifies human host factors crucial for influenza virus replication
}

\author{
Alexander Karlas ${ }^{1 *}$, Nikolaus Machuy ${ }^{1 *}$, Yujin Shin ${ }^{1}$, Klaus-Peter Pleissner ${ }^{2}$, Anita Artarini ${ }^{1}$, Dagmar Heuer ${ }^{1}$, \\ Daniel Becker ${ }^{1}$, Hany Khalil ${ }^{1}$, Lesley A. Ogilvie ${ }^{1}$, Simone Hess ${ }^{1} \uparrow$, André P. Mäurer ${ }^{1}$, Elke Müller ${ }^{1} \dagger$, Thorsten Wolff ${ }^{3}$, \\ Thomas Rudel $^{1} \dagger \&$ Thomas F. Meyer ${ }^{1}$
}

Influenza A virus, being responsible for seasonal epidemics and reoccurring pandemics, represents a worldwide threat to public health ${ }^{1}$. High mutation rates facilitate the generation of viral escape mutants, rendering vaccines and drugs directed against virusencoded targets potentially ineffective ${ }^{2}$. In contrast, targeting host cell determinants temporarily dispensable for the host but crucial for virus replication could prevent viral escape. Here we report the discovery of 287 human host cell genes influencing influenza A virus replication in a genome-wide RNA interference (RNAi) screen. Using an independent assay we confirmed 168 hits (59\%) inhibiting either the endemic H1N1 (119 hits) or the current pandemic swineorigin (121 hits) influenza A virus strains, with an overlap of $60 \%$. Notably, a subset of these common hits was also essential for replication of a highly pathogenic avian H5N1 strain. In-depth analyses of several factors provided insights into their infection stage relevance. Notably, SON DNA binding protein $(\mathrm{SON})^{3}$ was found to be important for normal trafficking of influenza virions to late endosomes early in infection. We also show that a small molecule inhibitor of CDC-like kinase $1(\mathrm{CLK} 1)^{4}$ reduces influenza virus replication by more than two orders of magnitude, an effect connected with impaired splicing of the viral M2 messenger RNA. Furthermore, influenza-virus-infected $p 27^{-1-}$ (cyclin-dependent kinase inhibitor 1B; Cdkn1b) mice accumulated significantly lower viral titres in the lung, providing in vivo evidence for the importance of this gene. Thus, our results highlight the potency of genome-wide RNAi screening for the dissection of virus-host interactions and the identification of drug targets for a broad range of influenza viruses.

During the course of infection, the influenza virus encounters numerous bottlenecks, constituted by host cell functions essential or inhibitory for viral propagation ${ }^{5}$. Comprehensive knowledge of such critical host cell determinants could provide valuable insight into the molecular mechanisms of viral replication and facilitate the development of a novel generation of drugs that target host cell factors and are thus less prone to select for resistant viral mutants. To identify host cell factors involved in the viral infection cycle in human cells, we conducted a genome-wide RNAi screen using a twostep approach (Fig. 1a). First, A549 human lung epithelial cells, transfected with small interfering RNAs $48 \mathrm{~h}$ before infection with influenza A H1N1 virus (A/WSN/33), were stained with a virusspecific antibody at $24 \mathrm{~h}$ after infection to monitor cell infection rates. Second, virus supernatants from these transfected A549 cells were transferred onto 293T human embryonic kidney reporter cells, containing an inducible influenza-virus-specific luciferase construct $(\text { FlaA })^{6}$. Assay reliability was confirmed with an siRNA directed

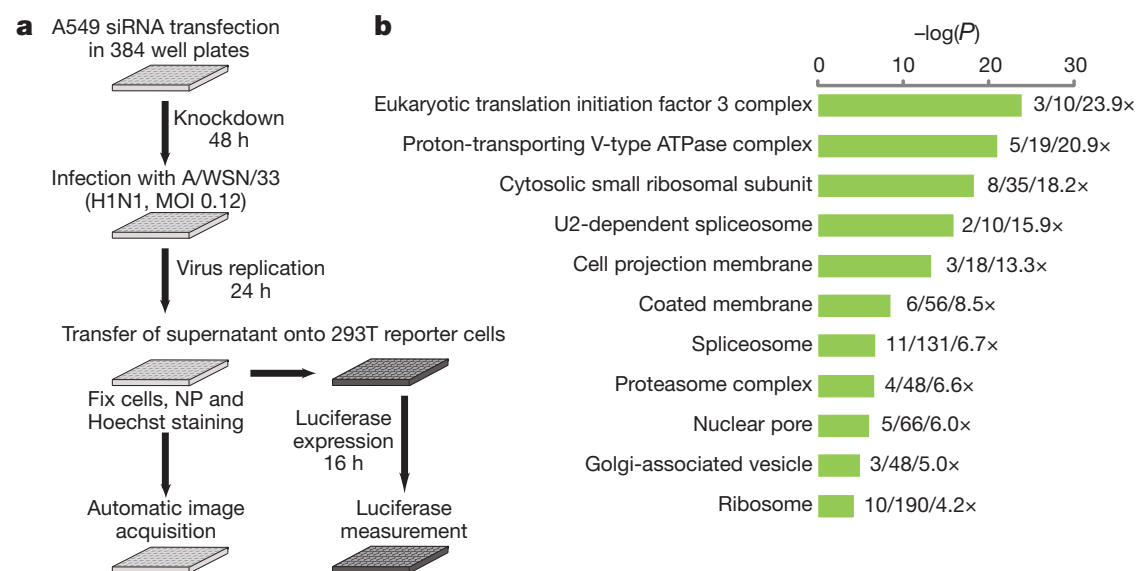

Figure 1 | Genome-wide RNAi screen reveals host factors required for the influenza infectious cycle. a, Outline of the screening procedures. MOI, multiplicity of infection. $\mathbf{b}$, Negative $\log (P$-values $)$ of enriched terms according to the GO of the cellular compartments. Numbers of identified factors per ontology, numbers of genes associated with the GO term, and the enrichment factors are indicated, respectively. c, Interaction among hits associated with RNA splicing, as assessed using the STRING interaction database. Green circles, primary hit; white circle, non-hit. Members of ribosomal and spliceosomal multi-protein complexes are enclosed in larger circles. Thick grey border indicates hits identified in Reactome analysis (see Supplementary Fig. 6).

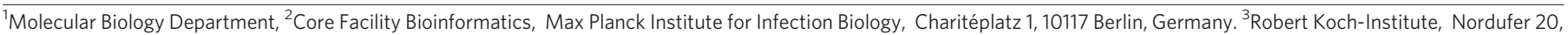

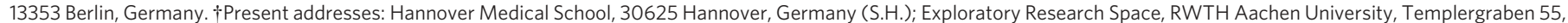
52056 Aachen, Germany (E.M.); Department of Microbiology, Biocenter, University of Würzburg, 97074 Würzburg, Germany (T.R.). 
against influenza virus nucleoprotein (NP) mRNA $\mathrm{m}^{7}$ Knockdown of NP effectively blocked viral replication, as assessed by immunofluorescence staining and the luciferase reporter assay (Supplementary Fig. 1). Statistical analyses further confirmed the robustness of our assay controls (NP and the non-targeting Allstars siRNA) and reproducibility of results (Supplementary Figs 2 and 3). Using this bipartite assay, we screened a genome-wide siRNA library consisting of approximately 62,000 siRNAs targeting $\sim 17,000$ annotated genes and $\sim 6,000$ predicted genes.

For identification of primary hits, three parameters were included: luciferase expression, the percentage of infected cells, as determined by immunofluorescence microscopy, and the total number of infected cells. After excluding non-expressed genes and toxic siRNAs, primary screening data from all three parameters were separately subjected to an analysis pipeline with statistical checkpoints at each step, finally leading to hit selection based on $Z$-scores below -2 (Supplementary Fig. 4 and Methods). Results from each of the three parameters were combined, and from a total of 22,843 human genes (annotated and predicted) 287 were designated primary hits (Supplementary Table 1).

Among these high-confidence candidates we found several genes known to have a pivotal role in influenza virus replication, for example, the nuclear export factors NXF1 (ref. 8) and XPO1 (ref. 9), as well as the vacuolar ATPase ATP6V0D1 (refs 10, 11). Gene ontology (GO) term enrichment analysis revealed that our data set was markedly enriched in gene categories associated with the proton-transporting two-sector ATPase complex, the spliceosome, the small ribosomal subunit, the eukaryotic translation initiation factor 3 (EIF3), the COPI coated vesicle transport and the nuclear pore complex (Fig. 1b and Supplementary Fig. 5), which comprise functional categories already associated with viral replication. Further bioinformatic analysis using Reactome ${ }^{12}$ corroborated the GO results (Supplementary Fig. 6). In-depth analysis of selected enriched functional categories using the STRING database revealed numerous interactions between factors associated with the same GO term (Supplementary Fig. 7). Interestingly, we found multiple factors connected with pre-mRNA splicing (Fig. 1c), which escaped detection in a previous RNAi screen using Drosophila cells ${ }^{13}$. However, the small ribosomal subunit and EIF3 were enriched in the Drosophila-based influenza screen ${ }^{13}$ but not in other viral RNAi screens ${ }^{14-17}$, indicating that these factors might be influenza-specific (see Supplementary Discussion $)^{18}$.

Next, we independently ascertained the significance of all 287 primary hits for replication of the influenza A/WSN/33 (H1N1) and the current pandemic swine-origin influenza A/Hamburg/04/ 2009 (H1N1) viruses. The number of viruses released from siRNAtransfected A549 cells was determined by titrating supernatants on Madin-Darby canine kidney (MDCK) cells. For each primary hit four different siRNAs were used individually to knockdown gene function. We found that 119 (A/WSN/33) and 121 (A/Hamburg/ $04 / 2009$ ) of the 287 primary hits decreased virus number by more than fivefold in comparison to control samples, with a least two siRNAs (Fig. 2a), without impairing cell viability (Supplementary Fig. 8). In total, 168 primary hits were validated, comprising an overall validation rate of $\sim 59 \%$. Notably, of the factors inhibiting viral replication, 72 were common to both influenza virus strains, indicative of their broad inhibitory potential (Fig. $2 b$ and Supplementary Table 2).

Validation was extended to the highly pathogenic avian-origin influenza A virus of the H5N1 subtype (A/Vietnam/1203/2004) using a subset of the common siRNAs (knockdown efficiencies shown in Supplementary Table 3). Strikingly, H5N1 virus replication decreased by more than two orders of magnitude using these siRNAs (Fig. 2c). Similarly, knockdown of identical targets inhibited replication of influenza A/WSN/33 (H1N1) virus and the pandemic A/Hamburg/ 04/2009 (H1N1) strain (Supplementary Fig. 9). The observation that a subset of common factors blocked replication of both swine- and avian-origin virus variants corroborates that these proteins constitute crucial sub-type-independent host-cell checkpoints.
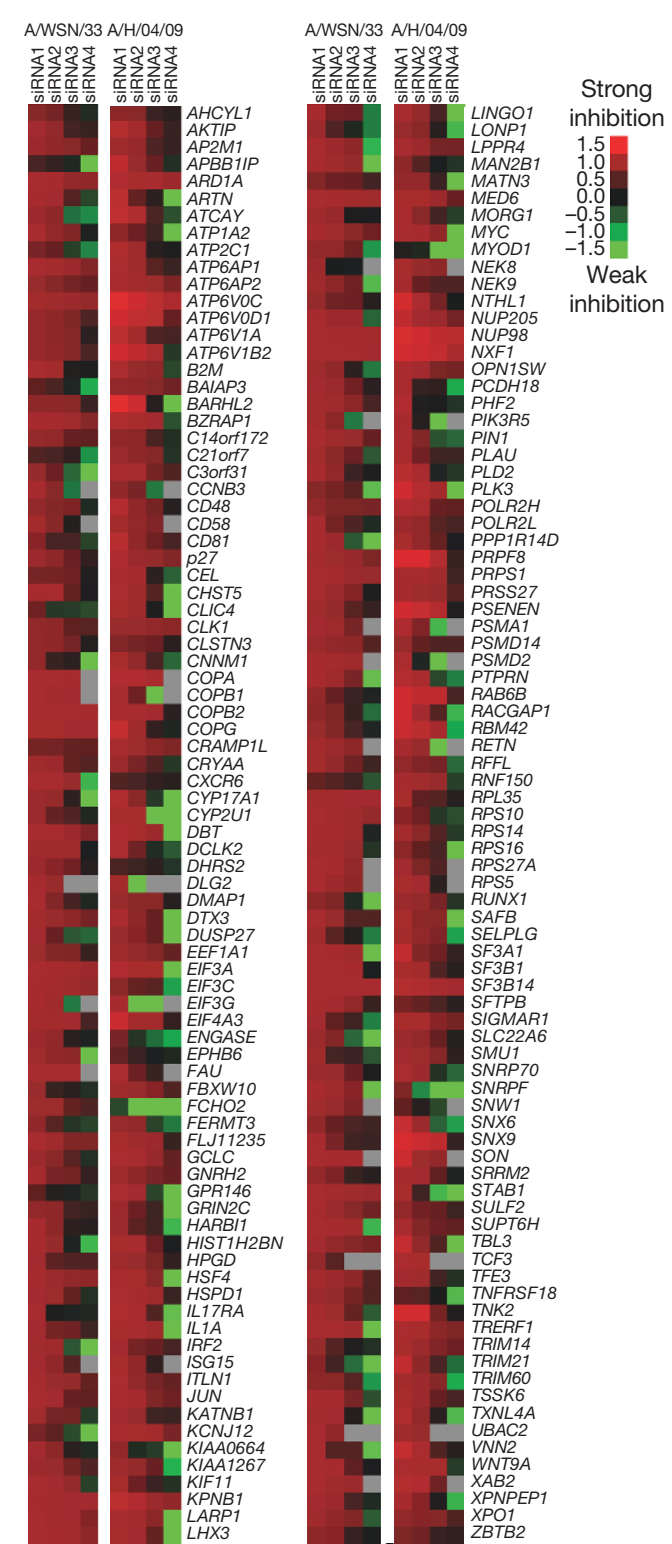

inhibition
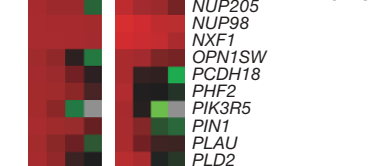

b

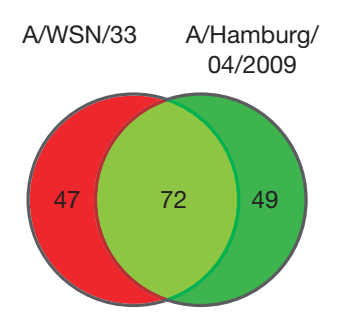

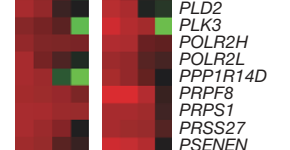
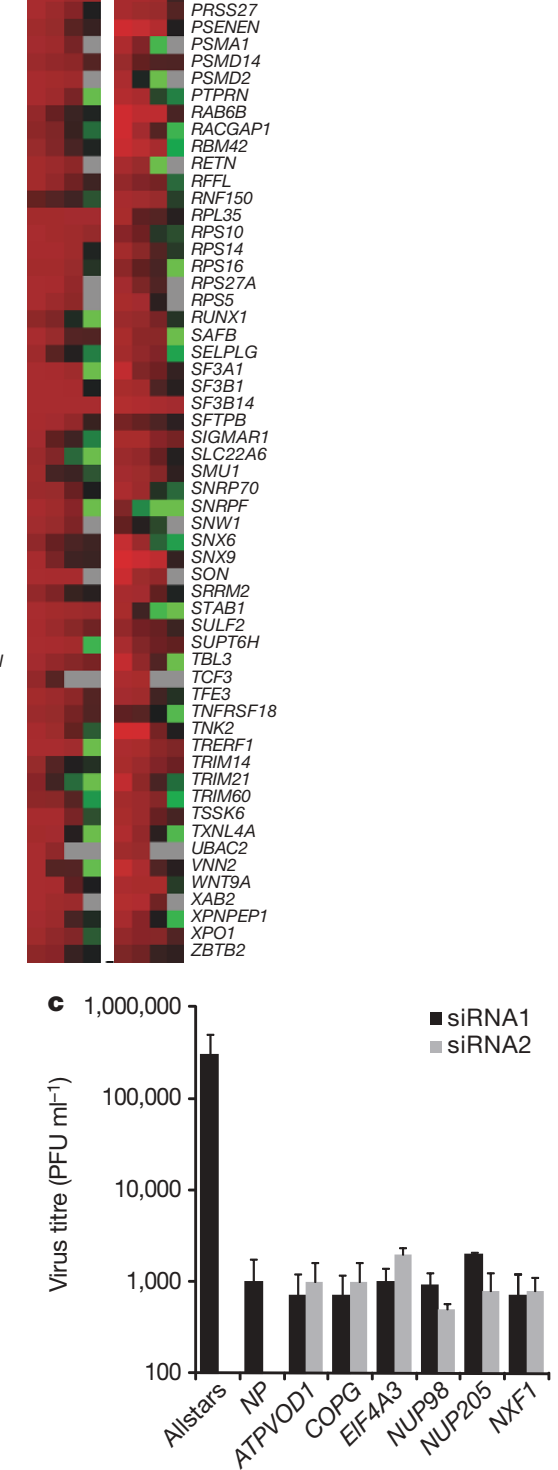

Figure 2 | Host cell factors affect replication of a broad range of influenza virus variants. a, Four siRNAs per gene were individually transfected in A549 cells followed by infection with influenza A/WSN/33 or A/Hamburg/ $04 / 2009$ viruses (both at an MOI of 0.001 ) in four independent experiments. Infectious viral particles were quantified at $48 \mathrm{~h}$ after infection using the replication assay and analysed by calculating the normalized percentage of inhibition. b, Venn diagram of hits validated in a. c, siRNAs (as indicated) were transfected in A549 cells and then infected ( $48 \mathrm{~h}$ later) with the avian H5N1 strain (A/Vietnam/1203/2004, MOI 0.1). Plaque-forming units (PFU) were quantified at $20 \mathrm{~h}$ after infection using the replication assay. Data show mean plus standard error of the mean (s.e.m.) of duplicate samples. 
The life-stage relevance of 18 targets, representing a variety of functional categories and affecting both $\mathrm{H} 1 \mathrm{~N} 1$ influenza viruses, was assessed by immunofluorescence staining for NP as a marker of viral ribonucleoprotein (vRNP) localization ${ }^{19}$. Typically, vRNP is confined to the nucleus early in infection, but enters the cytoplasm for packaging into progeny virions late in infection ${ }^{19}$. Here, upon knockdown of all targets, NP gave a mainly nuclear signal at $3 \mathrm{~h}$ after infection (Fig. 3a, upper panel), shifting towards cytoplasmic staining $2 \mathrm{~h}$ later (Fig. 3a, lower panel). In addition to the expected blockage of NP synthesis upon inhibition of NXF1 (ref. 8, 20), knockdown of several identified hits such as COPG, SON and ATP6VOC seemed to reduce NP expression levels (Fig. 3a, upper panel) and to delay export of NP from the nucleus (Fig. 3a, lower panel). Relative frequency distribution analysis of NP expression and cytosolic to nuclear NP ratios within single cells corroborated our microscopic observations (Supplementary Figs 10-12). In total, knockdown of 11 genes significantly reduced NP expression and interfered with nuclear export of NP.

To analyse the impact of targets-shown to affect NP synthesis and localization-on the synthesis of viral RNA, we infected siRNAtransfected cells with influenza virus and determined the levels of viral genomic RNA (vRNA) and viral mRNA at $2 \mathrm{~h}$ after infection by quantitative polymerase chain reaction with reverse transcription (qRTPCR $)^{7}$. Most of the analysed targets had no effect on virus cell entry, as indicated by robust vRNA detection (Fig. 3b). However, for many targets-including identified ATPases and SON, a protein known to repress hepatitis $B$ virion production ${ }^{3}$, plus several factors involved in RNA biogenesis, for example, NXF1—viral mRNA synthesis was substantially reduced (Fig. 3b). This demonstrates that virus propagation is affected at a stage between virus entry and mRNA synthesis.
Knockdown of SON also reduced vRNA levels (Fig. 3b), indicating that it functions in an infection step preceding viral mRNA synthesis. Accordingly, considerably fewer virus particles co-localized with CD63-labelled late endosomes upon SON knockdown (Fig. 3c; Supplementary Fig. 13), indicating that this factor is important for trafficking of influenza virions early in the infection cycle. Intriguingly, knockdown of the nucleoporin $98 \mathrm{kDa}$ (NUP98) increased vRNA level (Fig. 3b), probably due to accelerated de novo vRNA synthesis, but at the same time markedly decreased viral progeny (Fig. 2a and Supplementary Fig. 9). Consistent with its reported antiviral ${ }^{8}$ and proviral functions ${ }^{13}$, these seemingly contradictory results indicate that NUP98 exerts an inhibitory effect early in the life cycle but is mandatory for completion of viral replication. Taken together, these data reveal that the 11 targets (identified as reducing NP expression levels) interfere with early events in virus replication. In contrast, the remaining seven factors analysed in this set of experiments, such as CLK1 or p27 (CDKN1B), probably exert their function during later infection stages.

To mimic in vivo conditions more closely, we tested the effect of target knockdown on influenza virus replication in primary normal human bronchial epithelial cells (NHBE). Most notably, knockdown of CLK1 and ATP6V0D1 strongly reduced viral growth in these cells (Fig. 4a). We independently assessed the function of CLK1 by treating A549 cells with TG003, a chemical inhibitor of CLK1 (ref. 4). Influenza virus propagation was inhibited by more than 93\% (Fig. 4b) without exerting detectable toxic effects (Supplementary Fig. 14). CLK1 regulates alternative splicing in mammalian cells by phosphorylating the splicing factor SF2/ASF (refs 21, 22), therefore we hypothesized that inhibition of CLK1 would affect splicing of viral RNAs. In accordance, TG003 reduced levels of spliced M2 viral RNA,
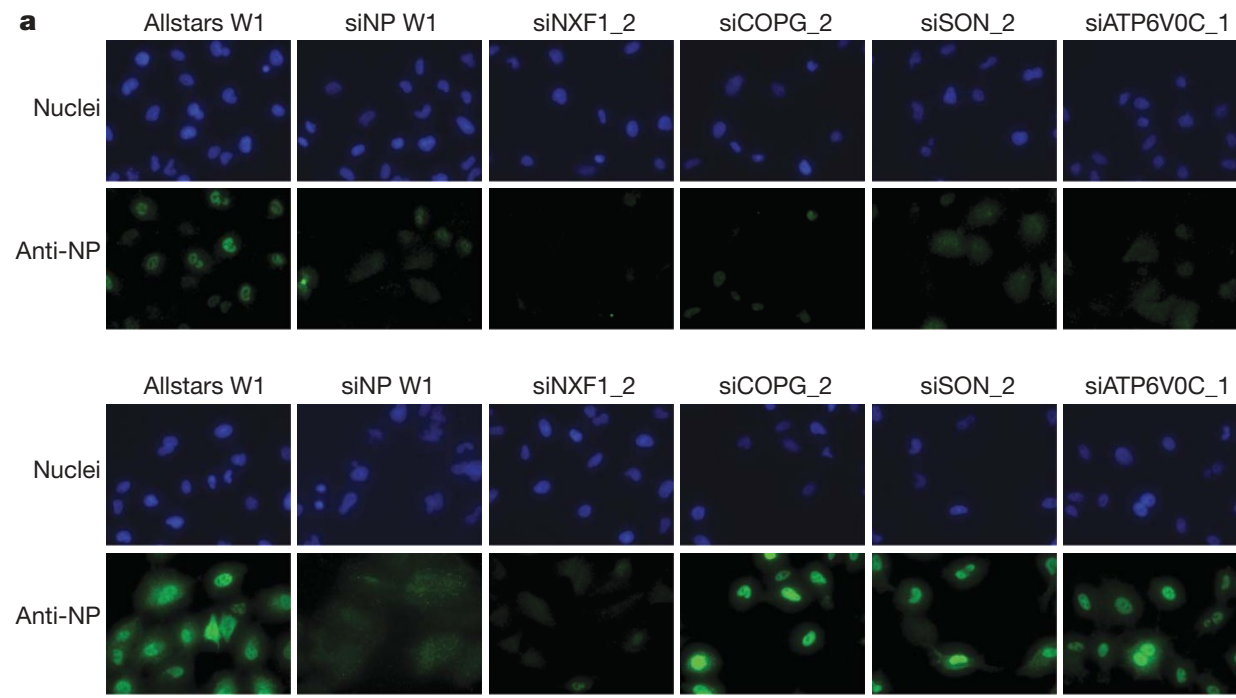

siNP W1
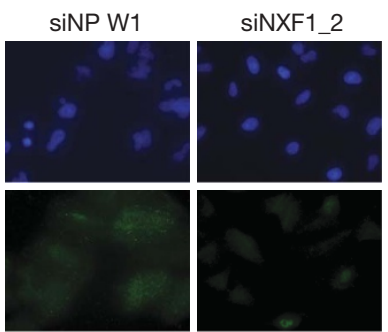

b
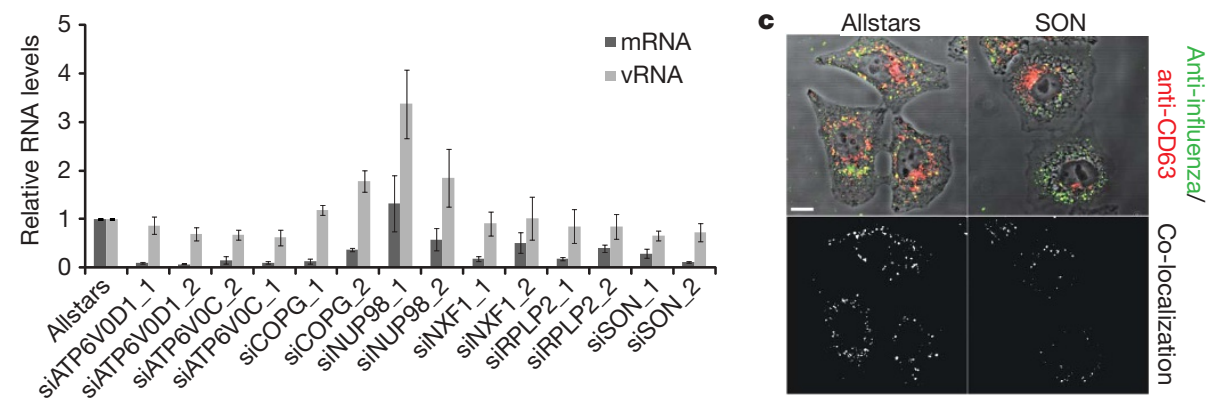

Figure 3 Dissection of infection processes affected by host cell factors. a, Transfected A549 cells were infected with influenza A/WSN/33 virus (MOI 5) for $3 \mathrm{~h}$ (upper panel) and $5 \mathrm{~h}$ (lower panel). Samples were stained for nuclei (blue) and NP (green). b, At $48 \mathrm{~h}$ after transfection, A549 cells were infected with influenza A/WSN/33 virus (MOI of 1 ). At $2 \mathrm{~h}$ after infection, vRNA and viral mRNA were quantified by qRT-PCR. RNA levels were normalized to the non-targeting (Allstars) siRNA control. Data show mean plus s.e.m. of three independent experiments. c, Transfected A549 cells were infected with influenza A/WSN/33 virus (MOI 10) for $45 \mathrm{~min}$. Samples were stained for influenza virus (green) and CD63 (red). Images are representative of three independent experiments in $\mathbf{a}$ and $\mathbf{c}$. 

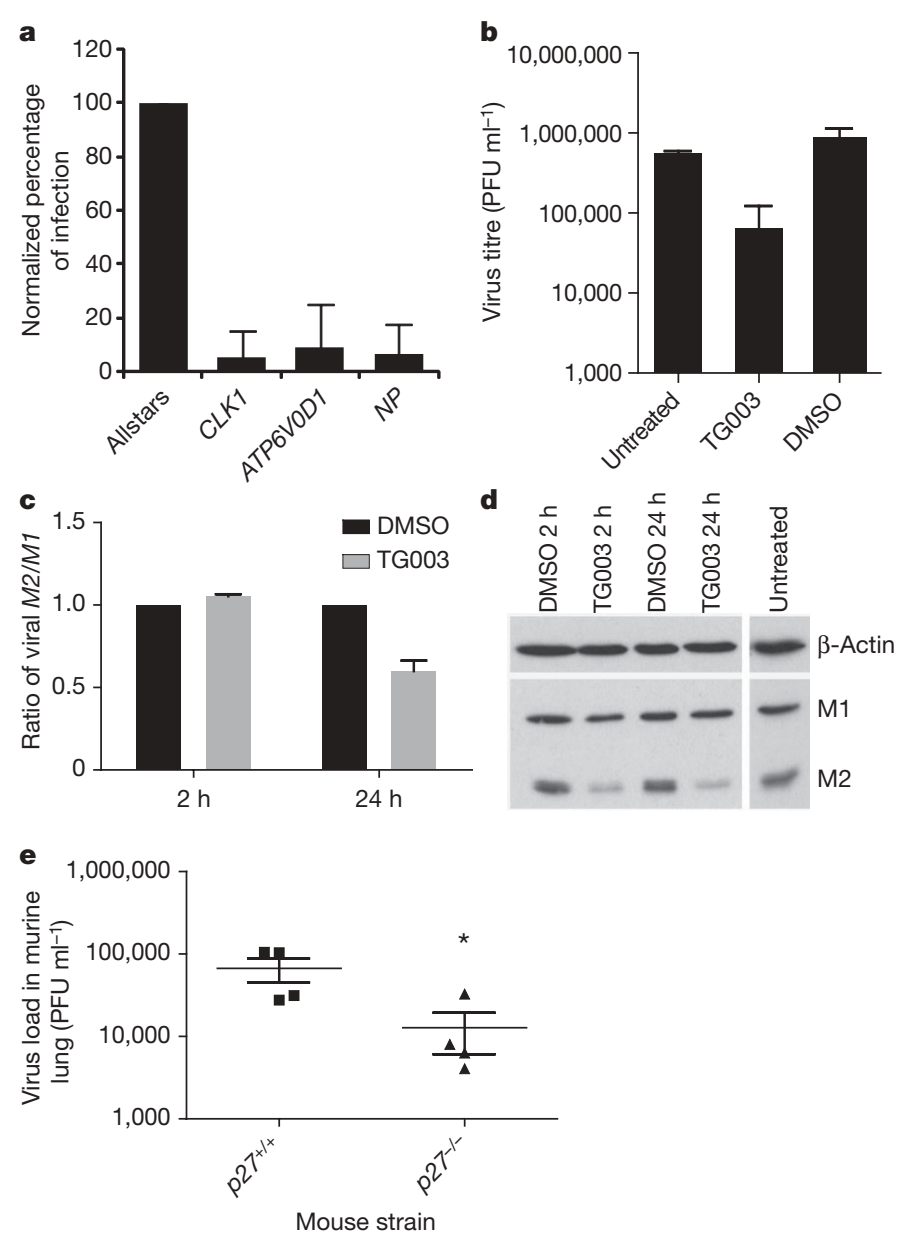

Figure 4 | In-depth analysis of the impact of p27 and CLK1 on influenza A virus infection. a, Quantification of virus replication in primary NHBE cells after siRNA-mediated target knockdown using the replication assay. Cells were infected with influenza A/WSN/33 virus (MOI 0.1) $48 \mathrm{~h}$ after transfection. b, A549 cells were pre-treated with TG003 $(50 \mu \mathrm{M})$ or dimethylsulphoxide (DMSO) for $24 \mathrm{~h}$ and subsequently infected with influenza $\mathrm{A} / \mathrm{WSN} / 33$ virus (MOI 0.01). Infectious viral particles were quantified at $40 \mathrm{~h}$ after infection. c, d, RNA ratio (c) and protein levels (d) of spliced M2 and M1 after inhibition of CLK1 by TG003. A549 cells were pretreated for $2 \mathrm{~h}$ or $24 \mathrm{~h}$ with TG003 $(50 \mu \mathrm{M})$ or DMSO, then infected with influenza A/WSN/33 virus (MOI 4) for $5 \mathrm{~h}$. e, C57BL/6 wild-type or homozygous $p 27^{-1-}$ mice $(n=4)$ were intranasally infected with influenza A/Puerto Rico/8/34 virus $(10 \times \mathrm{LD} 50)$ and at $48 \mathrm{~h}$ after infection infectious viral particles within the lungs were quantified. Student's $t$-test was used to determine the $P$ value; asterisk, $P=0.041$. Data in a-c are mean plus standard deviation (s.d.) of three independent experiments. Blots in $\mathbf{d}$ are representative of three independent experiments.

whereas unspliced M1 and NS1/NS2 were unaffected (Fig. 4c, data not shown). Immunoblot analysis corroborated our qRT-PCR results, revealing markedly reduced M2 protein levels following treatment with TG003, whereas M1 protein levels remained relatively constant (Fig. 4d). Because the SF2/ASF complex is important for splicing and the shuttling of spliced viral mRNAs to the cytoplasm ${ }^{23}$, it is conceivable that reduction of M2 protein expression was at least partially caused by nuclear retention of its mRNA transcript. Our finding that CLK1 is involved in processing viral M2 mRNA is consistent with the essential role of the SF2/ASF splicing factor in viral M2 ion channel protein production ${ }^{24}$. Interestingly, replication of vesicular stomatitis virus (VSV), which, unlike influenza, does not depend on splicing of its own viral RNA, was only slightly reduced in the presence of TG003 (Supplementary Fig. 15).

During the primary screen and the hit validation, knockdown of the cell cycle regulator p27 led to a strong inhibition of influenza virus replication. To confirm the impact of p27 on viral replication under in vivo conditions, $p 27^{-1-}$ mice were intranasally infected with influenza A/Puerto Rico/8/34 (H1N1) virus. At $2 \mathrm{~d}$ after infection, virus load within the lungs of $p 27^{-1-}$ mice was significantly reduced (Fig. 4e). The observation that a lack of p27 reduces influenza virus replication in vivo but does not affect mouse viability indicates that certain cellular proteins involved in influenza virus replication are dispensable for the host organism.

This genome-wide RNAi screen in human cells for factors affecting influenza virus replication has provided new and comprehensive information on host cell determinants of replication, and uncovered potential targets for novel antiviral strategies. We provide in vitro and in vivo evidence for the role of CLK1 and the tumour suppressor p27, using a small molecule inhibitor and a homozygous knockout model, respectively. Most of the hits analysed in-depth seem to function during early infection processes such as viral protein synthesis and nuclear export of viral RNA. Importantly, most of the validated hits are essential for a broad spectrum of influenza viruses, including the pandemic swine-origin H1N1 influenza virus and even a highly pathogenic avian H5N1 strain. This holds promise for the therapeutic potential of these targets against novel emerging influenza viruses with minimized likelihood of developing drug-resistant variants. Transient interference with distinct host cell functions during infection is likely to extend our current armament, consisting of vaccines and virus-targeted drugs, in the battle against the recurring threat of seasonal and pandemic influenza virus infections.

\section{METHODS SUMMARY}

siRNA screening. All siRNAs ( $4 \mu \mathrm{l}$ per well, $200 \mathrm{nM}$ ) were arrayed in 384 -well plates. To each well, $8 \mu \mathrm{l}$ of RPMI medium (Invitrogen) containing $0.35 \mu \mathrm{l}$ HiperFect (Qiagen) was added and plates were shaken for $1 \mathrm{~min}$. After $10 \mathrm{~min}$ incubation at room temperature, a cell suspension $(28 \mu \mathrm{l})$ of 500 cells was added to give a final siRNA concentration of $20 \mathrm{nM}$. Cells were incubated at $37^{\circ} \mathrm{C}$ and $5 \% \mathrm{CO}_{2}$ for $48 \mathrm{~h}$ before infection at MOI 0.12 . At $24 \mathrm{~h}$ after infection, supernatants were transferred onto freshly seeded 293T reporter cells, incubated for $16 \mathrm{~h}$ at $37^{\circ} \mathrm{C}$ and $5 \% \mathrm{CO}_{2}$ and then luciferase activities were measured. The A549 cells were fixed, stained for nuclei and NP, and analysed using the Acumen eX3 Cytometer (TTP Labtech). All multiwell pipetting steps were performed using a Biomek FX ${ }^{\mathrm{P}}$ Laboratory Automation Workstation (Beckman Coulter). An siRNA library (Qiagen Hu_Genome 1.0 and Human Druggable Genome siRNA Set V2.0; Qiagen) containing four siRNAs per gene for the druggable genome $^{25}$ and two siRNAs per gene for non-druggable and predicted genes was screened three times independently. The following siRNAs with the indicated target sequence were included in all screening plates as controls: siNP 5'-AAGGAUCUUAUUUCUUCGGAG-3', siPLK1 5'-CACCATATGAATTGT ACAGAA-3' and Allstars (Qiagen).

Full Methods and any associated references are available in the online version of the paper at www.nature.com/nature.

\section{Received 10 October; accepted 17 December 2009.}

Published online 17 January 2010.

1. Horimoto, T. \& Kawaoka, Y. Influenza: lessons from past pandemics, warnings from current incidents. Nature Rev. Microbiol. 3, 591-600 (2005).

2. Neumann, G., Noda, T. \& Kawaoka, Y. Emergence and pandemic potential of swine-origin H1N1 influenza virus. Nature 459, 931-939 (2009).

3. Sun, C. T. et al. Transcription repression of human hepatitis B virus genes by negative regulatory element-binding protein/SON. J. Biol. Chem. 276, 24059-24067 (2001).

4. Muraki, M. et al. Manipulation of alternative splicing by a newly developed inhibitor of Clks. J. Biol. Chem. 279, 24246-24254 (2004).

5. Ludwig, L., Planz, O., Pleschka, S. \& Wolff, T. Influenza-virus-induced signaling cascades: targets for antiviral therapy? Trends Mol. Med. 9, 46-52 (2003).

6. Lutz, A., Dyall, J., Olivo, P. D. \& Pekosz, A. Virus-inducible reporter genes as a tool for detecting and quantifying influenza A virus replication. J. Virol. Methods 126, 13-20 (2005).

7. $\mathrm{Ge}, \mathrm{Q}$. et al. RNA interference of influenza virus production by directly targeting mRNA for degradation and indirectly inhibiting all viral RNA transcription. Proc. Natl Acad. Sci. USA 100, 2718-2723 (2003).

8. Satterly, N. et al. Influenza virus targets the mRNA export machinery and the nuclear pore complex. Proc. Natl Acad. Sci. USA 104, 1853-1858 (2007).

9. Elton, E. et al. Interaction of the influenza virus nucleoprotein with the cellular CRM1-mediated nuclear export pathway. J. Virol. 75, 408-419 (2001).

10. Guinea, R. \& Carrasco, L. Requirement for vacuolar proton-ATPase activity during entry of influenza virus into cells. J. Virol. 69, 2306-2312 (1995) 
11. Perez, L. \& Carrasco, L. Involvement of the vacuolar $\mathrm{H}^{+}$-ATPase in animal virus entry. J. Gen. Virol. 75, 2595-2606 (1994).

12. Joshi-Tope, G. et al. Reactome: a knowledgebase of biological pathways. Nucleic Acids Res. 33, D428-D432 (2005).

13. Hao, L. et al. Drosophila RNAi screen identifies host genes important for influenza virus replication. Nature 454, 890-893 (2008)

14. Krishnan, M. N. et al. RNA interference screen for human genes associated with West Nile virus infection. Nature 455, 242-245 (2008)

15. Sessions, O. M. et al. Discovery of insect and human dengue virus host factors. 458, 1047-1050 (2009).

16. Brass, A. L. et al. Identification of host proteins required for HIV infection through a functional genomic screen. Science 319, 921-926 (2008).

17. Konig, R. et al. Global analysis of host-pathogen interactions that regulate earlystage HIV-1 replication. Cell 135, 49-60 (2008).

18. Cherry, S. et al. Genome-wide RNAi screen reveals a specific sensitivity of IREScontaining RNA viruses to host translation inhibition. Genes Dev. 19, 445-452 (2005)

19. Josset, L., Frobert, E. \& Rosa-Calatrava, M. Influenza A replication and host nuclear compartments: many changes and many questions. J. Clin. Virol. 43, 381-390 (2008).

20. Cullen, B. R. Nuclear mRNA export: insights from virology. Trends Biochem. Sci. 28, 419-424 (2003).

21. Bullock, A. N. et al. Kinase domain insertions define distinct roles of CLK kinases in SR protein phosphorylation. Structure 17, 352-362 (2009).

22. Hagiwara, M. Alternative splicing: a new drug target of the post-genome era. Biochim. Biophys. Acta 1754, 324-331 (2005).

23. Huang, Y., Yario, T. A. \& Steitz, J. A. A molecular link between SR protein dephosphorylation and mRNA export. Proc. Natl Acad. Sci. USA 101, 9666-9670 (2004).
24. Shih, S. R. \& Krug, R. M. Novel exploitation of a nuclear function by influenza virus: the cellular SF2/ASF splicing factor controls the amount of the essential viral M2 ion channel protein in infected cells. EMBO J. 15, 5415-5427 (1996).

25. Hopkins, A. L. \& Groom, C. R. The druggable genome. Nature Rev. Drug Discov. 1, 727-730 (2002)

Supplementary Information is linked to the online version of the paper at www.nature.com/nature.

Acknowledgements The authors would like to thank M. Drabkina, G. Heins, D. Khalil and D. Manntz for technical support, and K. Hoffmann and the experimental animal facility, headed by U. Klemm, for supporting mouse experiments. We also thank A. Pekosz for providing the influenza luciferase reporter construct and S. Becker for the pandemic H1N1 influenza strain. This work was supported through the EU FP6 project RIGHT (LSHB-CT-2004-005276), ERA-Net Pathogenomics (grant no. 0313938A) and a grant from FCI to T.F.M., and the RiNA network Berlin to T.R. and T.F.M. T.W. acknowledges German Government support via BMG (FSI program) and BMBF (FluResearchNet). H.K. and A.A. are student fellows of the Egyptian Government and IMPRS-IDI, respectively.

Author Contributions A.K., N.M. and T.F.M. conceived the project; A.K., N.M., T.W. and T.F.M. designed experiments; A.K., N.M., Y.S., A.A., D.H., D.B., H.K. and T.W. performed experiments; A.K., N.M., K.-P.P., S.H. and A.P.M. conducted bioinformatics; N.M., E.M., T.R. and T.F.M. established the screening platform; A.K., N.M., D.H., D.B., L.A.O. and T.F.M. wrote the manuscript.

Author Information Reprints and permissions information is available at www.nature.com/reprints. The authors declare no competing financial interests. Correspondence and requests for materials should be addressed to T.F.M. (meyer@mpiib-berlin.mpg.de). 


\section{METHODS}

Cells and viruses. The A549 human lung epithelial cell line (CCL-185, ATCCLGC) was grown in DMEM media (Invitrogen) supplemented with $4 \mathrm{mM}$ L-glutamine, $4 \mathrm{mM}$ sodium pyruvate, $100 \mathrm{U} \mathrm{ml}^{-1}$ penicillin/streptomycin and $10 \%$ fetal calf serum (FCS, Biochrome) (DMEM complete medium) at $37^{\circ} \mathrm{C}$ and $5 \% \mathrm{CO}_{2}$. The human embryonic kidney cell line 293T (CRL-11268, ATCC-LGC) and the Madin-Darby canine kidney cells (MDCK, CCL-34, ATCC-LGC) were grown in DMEM supplemented with $4 \mathrm{mM} \mathrm{L-glutamine,} 100 \mathrm{U} \mathrm{ml}^{-1}$ penicillin/ streptomycin and 10\% FCS. Primary normal human bronchial epithelial cells (NHBE, CC-2541, Lonza) were grown in Clonetics BEGM BulletKit (CC-3170, Lonza) supplemented with the following growth supplements: BPE, hydrocortisone, hEGF, epinephrine, transferrin, insulin, retinoic acid, triiodothyronine, GA-1000. Supplements added at $0.5 \mathrm{ml}$ per $500 \mathrm{ml}$ medium, except BPE $(2 \mathrm{ml}$ per $500 \mathrm{ml})$. Cells were regularly checked for mycoplasma contamination by PCR. The influenza virus strains A/WSN/33 (H1N1) and A/Puerto Rico/8/34 (H1N1) were grown in the allantoic cavities of 11-day-old embryonated chicken eggs. Production of recombinant highly pathogenic influenza A/Vietnam/1203/ 2004 virus (H5N1) by reverse genetics was done essentially as described previously $^{26}$. The pandemic H1N1 A/Hamburg/04/2009 strain was provided by S. Becker and was propagated in MDCK cells in DMEM supplemented with $1 \mu \mathrm{g}$ trypsin per $\mathrm{ml}$ in the absence of FCS. Virus stocks were titrated by standard plaque assay on MDCK cells using an agar overlay medium ${ }^{27}$.

siRNA screening. All siRNAs ( $4 \mu \mathrm{l}$ per well; $200 \mathrm{nM}$ ) were arrayed in 384-well plates. To each well, $8 \mu$ l of RPMI medium (Invitrogen) containing $0.35 \mu \mathrm{l}$ HiperFect (Qiagen) was added and plates were shaken for $1 \mathrm{~min}$. After $10 \mathrm{~min}$ incubation at room temperature, a cell suspension $(28 \mu \mathrm{l})$ containing 500 cells was added to give a final siRNA concentration of $20 \mathrm{nM}$. Cells were incubated at $37^{\circ} \mathrm{C}$ and $5 \% \mathrm{CO}_{2}$ for $48 \mathrm{~h}$ before infection at an MOI of 0.12 (see below). At $24 \mathrm{~h}$ after infection, supernatants were transferred onto freshly seeded 293T reporter cells, incubated for $16 \mathrm{~h}$ at $37^{\circ} \mathrm{C}$ and $5 \% \mathrm{CO}_{2}$ and then luciferase activities were measured (see below). The A549 cells were fixed, stained for nuclei and NP, and analysed using the Acumen eX3 Cytometer (TTP Labtech). The number of automatically counted nuclei was further used to estimate cytotoxic effects of specific siRNAs. The siRNA was classified as being toxic if 750 or fewer nuclei were determined within one well of a 384 -well plate. All multiwell pipetting steps were performed using a Biomek $\mathrm{FX}^{\mathrm{P}}$ Laboratory Automation Workstation (Beckman Coulter). An siRNA library (Qiagen Hu_Genome 1.0 and Human Druggable Genome siRNA Set V2.0; Qiagen) containing four siRNAs per gene for the druggable genome $e^{25}$ and two siRNAs per gene for non-druggable and predicted genes was screened three times independently. The following siRNAs with the indicated target sequence were included in all screening plates as controls: siNP 5'-AAGGAUCUUAUUUCUUCGGAG-3', siPLK1 5'-CACCATAT GAATTGTACAGAA- $3^{\prime}$ and Allstars (Qiagen).

Luciferase reporter assay. To quantify infectious viruses in the supernatants of siRNA-transfected A549 cells during the primary RNAi screen, we used a luciferasebased reporter system. 293T cells were transfected in batches with a FluA luc plasmid $^{6}$, one day later seeded into 384-well plates at concentrations of $1 \times 10^{4}$ per well, and subsequently infected with $12.5 \mu \mathrm{l}$ of virus-containing supernatant. At $16 \mathrm{~h}$ after infection, Bright-Glo firefly luciferase substrate (Promega) was added and luciferase activities in cell lysates were measured using the Envision multilabel plate reader (PerkinElmer). Transfection of 239T cells with the influenza-virus-specific luciferase construct (FlaA) induces expression of firefly luciferase transcripts flanked by the untranslated region of the influenza A/WSN/33 virus nucleoprotein (NP) segment. Luciferase expression is therefore only detectable in the presence of the viral polymerase, thus allowing quantification of infectious viruses.

siRNA transfection for validation experiments in 96- and 12-well plates. All siRNAs were purchased from Qiagen. For siRNA transfection of A549 cells in 96-well plates, $20 \mu \mathrm{l}$ of a $100 \mathrm{nM}$ siRNA dilution in DMEM without supplements was mixed with $1 \mu \mathrm{l}$ HiperFect plus $9 \mu$ DMEM medium and incubated for $10 \mathrm{~min}$ at room temperature. Complex formation was stopped by addition of $25 \mu \mathrm{l}$ DMEM complete medium. Next, 3,000 A549 cells in $50 \mu \mathrm{l}$ DMEM complete medium were seeded into each well and incubated at $37^{\circ} \mathrm{C}$ and $5 \% \mathrm{CO}_{2}$ for the indicated time periods. For siRNA transfection of NHBE cells in 96-well plates, BEGM medium (with/without supplements) was used and 15,000 cells per well were seeded. For western blot experiments, siRNA transfection was carried out in 12 -well plates. For each well, $1 \mu \mathrm{l}$ of a $20 \mu \mathrm{M}$ siRNA solution was diluted in $99 \mu \mathrm{l}$ RPMI (Invitrogen) supplemented with $25 \mathrm{mM}$ HEPES (Invitrogen). The mix was incubated at room temperature for $5 \mathrm{~min}$ before addition of $5 \mu \mathrm{l} \mathrm{HiperFect}$ (Qiagen) and further $15 \mathrm{~min}$ incubation at room temperature. Each complex was added to 50,000 A549 cells in $900 \mu$ DMEM complete medium, mixed carefully, and then transferred to 12 -well plates. After $6 \mathrm{~h}$ incubation at $37^{\circ} \mathrm{C}$ and $5 \% \mathrm{CO}_{2}$, the medium was exchanged for fresh DMEM complete medium and the cells were incubated for an additional $48 \mathrm{~h}$ using the same growth conditions.
Indirect immunofluorescence labelling. Cells were fixed with 3.7\% formaldehyde and permeabilized with $0.3 \%$ Triton X-100, $10 \%$ FCS in PBS. Samples were sequentially incubated with a primary antibody against the viral nucleoprotein (NP, clone AA5H, AbD Serotec) diluted 1:10,000 in PBS with 10\% FCS, $0.1 \%$ Tween 20 for $1 \mathrm{~h}$ at room temperature, followed by an incubation with the secondary Cy3-conjugated antibody directed against mouse IgG (1:100 in PBS with $10 \%$ FCS, $0.1 \%$ Tween 20 and $0.1 \%$ Hoechst dye used to stain cellular DNA). Numbers of infected versus non-infected cells were determined using automated microscopy (Olympus, Soft Imaging Solutions) or, for the primary siRNA screen, the Acumen eX3 microplate cytometer (TTP LabTech).

Automated microscopy and image analysis. The numbers of influenza-infected and host cells were determined using an automated microscope (Olympus Soft Imaging Solutions). Images were taken with DAPI and Cy3 filter sets (AHFAnalysetechnik). Scan ${ }^{\mathrm{R}}$ Analysis Software (Olympus Soft Imaging Solutions) was used to automatically identify and quantify influenza nuclear protein (NP) and cell nuclei. For determination of NP localization, mean and total intensities of NP were analysed. NP located within the same area as the Hoechst staining was defined as nuclear NP. NP located within a 5-pixel-wide ring around the nuclei was defined as cytosolic NP. The distance between the inner edge of the ring and the nuclei was set at 1 pixel. For each experiment identical camera settings were used.

Host cell viability determination by WST-1 assay. Determination of host cell viability upon siRNA transfection was performed using cell proliferation assay WST-1 (Roche). WST-1 reagent was added to the cells $48 \mathrm{~h}$ after siRNA transfection and incubated at $37^{\circ} \mathrm{C}$ for $1.5 \mathrm{~h}$. Absorbance was measured at $460 \mathrm{~nm}$ and at the reference wavelength $590 \mathrm{~nm}$. Non-targeting siRNA Allstars and siPLK1 were used as a positive and negative control, respectively.

Virus infection. Cells were washed with PBS and then infected with influenza at the indicated MOIs in infection buffer (PBS supplemented with $0.2 \%$ bovine serum albumin) for $60 \mathrm{~min}$ at room temperature. Cells were washed again (in infection buffer) and incubated for the indicated time periods at $37^{\circ} \mathrm{C}$ in DMEM supplemented with $0.2 \%$ bovine serum albumin, $4 \mathrm{mM}$ L-glutamine and antibiotics (A549) or BEGM with supplements (NHBE), unless otherwise stated. All infection experiments with $\mathrm{A} / \mathrm{WSN} / 33, \mathrm{~A} / \mathrm{Puerto} \mathrm{Rico} / 8 / 34$ and with A/Hamburg/04/2009 H1N1 viruses were performed under biosafety level (BSL) 2 conditions, whereas BSL 3 conditions were used for experiments with A/Vietnam/1203/2004 (H5N1).

Replication assay. To quantify infectious virus particles in infected cell culture supernatants, 5,000 or 12,000 MDCK cells were seeded in 384- or 96-well plates, respectively. One day later the cells were washed twice, infected with a dilution series of cell culture supernatants and incubated at room temperature for $1 \mathrm{~h}$. Infection buffer (as above) was added ( $40 \mu \mathrm{l}$ or $100 \mu \mathrm{l}$ per well) and plates were incubated at $37^{\circ} \mathrm{C}, 5 \% \mathrm{CO}_{2}$ for $6 \mathrm{~h}$, followed by fixation with $3.7 \%$ formaldehyde, antibody staining and automatic image processing, as described in 'Indirect immunofluorescence labelling'.

Gene enrichment and network analysis. For gene enrichment analysis, we modified the R-script available from the Gaggle website (http://gaggle.systemsbiology. net/svn/gaggle/PIPE2.0/trunk/PIPEletResourceDir/GOTableEnrichment/ GOEnrichmentScript.R). This script applies the R-package GOstats developed by ref. 28 and is available at the Bioconductor website (http://www.bioconductor. org). Briefly, we defined a gene universe consisting of 22,843 genes contained and annotated in the genome-wide library and processed the hit list against this universe with respect to molecular function (MF), cellular component (CC) and biological process (BP). Each Gene Ontology term is associated with $X$ number of genes, providing a relative frequency $A$. In the hit list, the same term is connected to $Y$ genes giving a relative frequency $B . B$ divided by $A$ is the enrichment factor.

The 287 'high-confidence' hits were also uploaded as gene identifiers using the Sky-Painter tool of the Reactome website (http://www.reactome.org). Significant events calculated by the application's Fisher's exact test were identified and coloured accordingly. Network analysis was carried out using the STRING database (http://string.embl.de/).

Confocal microscopy. Fusion between influenza viruses and cellular endosomes was detected using confocal microscopy. A549 cells were plated onto coverslips in 12 -well plates at a density of $5 \times 10^{4}$ cells per well and directly transfected in suspension with indicated siRNAs, followed by infection with influenza A/WSN/ 33 virus (MOI 10) $48 \mathrm{~h}$ after transfection. During the infection process, cells were kept on ice for $45 \mathrm{~min}$, washed twice with cold infection buffer (see above) and subsequently incubated with pre-warmed infection media (DMEM supplemented with $0.2 \%$ bovine serum albumin, $4 \mathrm{mM}$ L-glutamine and antibiotics). After 15, 45 and 90 min cells were fixed with $4 \%$ paraformaldehyde and permeabilized for 20 min with $0.2 \%$ BSA in PBS and $0.2 \%$ Triton X-100. Cells were then incubated for $1 \mathrm{~h}$ with antibodies targeting CD63 (Millipore) at a dilution of 1:70 and a polyclonal serum against influenza $(1: 1,000)$, followed by incubation with a fluorescently labelled secondary antibody (dilution 1:100). Samples were mounted in 
MOWIOL. Images were taken with a Leica TCS-SP confocal microscope and processed using Adobe Photoshop 11.0.

Immunoblotting. For immunoblotting, cells were washed with PBS and lysed in $1 \times$ SDS sample buffer containing $75 \mathrm{mM}$ Tris $\mathrm{HCl}$ ( $\mathrm{pH} 6.8$ ), $25 \%$ glycerol, $0.6 \%$ SDS, $7.5 \% \beta$-mercaptoethanol and $0.001 \%$ bromophenol blue. Protein lysates $(20 \mu \mathrm{l})$ were loaded and separated on a 10\% SDS-polyacrylamide gel. Separated proteins were transferred to a PVDF membrane and detected using mouse monoclonal antibodies against viral matrix protein (M1, AbD Serotec), viral ion channel protein (M2, Santa Cruz) or $\beta$-actin (Sigma) at 1:100, 1:10,00 or 1:2,500 dilution, respectively, followed by incubation with a secondary sheep anti-mouse IgG horseradish peroxidase $(1: 10,000)$. Staining was performed with ECL western blotting detection reagent (Amersham). $\beta$-Actin was used as a loading control. Band intensities were determined using the Aida image analyser (V.4.03) (2D/Densitometry) and normalized to $\beta$-actin.

Quantitative RT-PCR. For the detection of viral RNA (vRNA) or viral mRNA, quantitative RT-PCR (qRT-PCR) was performed as previously described ${ }^{7}$. Briefly, A549 cells infected with influenza A/WSN/33 virus (MOI 1) were lysed with RLT lysis buffer (Qiagen). For reverse transcription of viral mRNA, an oligo(dT) 18 primer was used: the negative stranded vRNA of the gene segment PA was converted to CDNA using a PA-specific oligonucleotide (5' - GCTTCTT ATCGTTCAGGCTCTTAGG-3'). Resulting cDNAs were quantified by qRTPCR with oligonucleotides specific for PA (5' - GCTTCTTATCGTTCAGGCT CTTAGG-3' and 5' - CCGAGAAGCATTAAGCAAAACCCAG-3'). GAPDH was amplified using the oligonucleotides GAPDH for 5'-GGTATCGTGGAAGGA CTCATGAC-3'; GAPDH_rev 5'-ATGCCAGTGAGCTTCCCGTTCAG-3'. Levels of GAPDH were used for normalization. All experiments were done in triplicate.

To quantify the levels of spliced and unspliced mRNAs, infection of A549 cells with influenza A/WSN/33 virus was performed at an MOI of 4 for $5 \mathrm{~h}$. RNA was then isolated using the RNeasy Mini Kit (Qiagen) and treated with DNase (Ambion) according to manufacturer's instructions. Reverse transcription of viral mRNA was performed using oligo(dT) primer and the synthesized cDNA was subjected to real-time PCR using primers specific for M1 (5'-GACC AATCCTGTCACCTC-3' and $5^{\prime}$-GATCTCCGTTCCCATTAAGAG-3') and M2 (5'-GAGGTCGAAACGCCTAT- ${ }^{\prime}$ and $5^{\prime}$-CTCCAGCTCTATGTTGACA AA- $\left.3^{\prime}\right)$, as described previously ${ }^{29}$. Levels of $M 1$ and $M 2$ mRNA were normalized to GAPDH.

Validation of RNAi by quantitative PCR. siRNA validation was performed as previously described ${ }^{30}$. Briefly, one day before transfection, 3,000 cells per well were seeded onto a 96-well plate. Transfection was performed with a final siRNA concentration of $56 \mathrm{nM}$ with $0.25 \mu \mathrm{l}$ HiPerFect (Qiagen). Knockdown measurements were performed independently three times. After $48 \mathrm{~h}$, RNA was isolated using the RNeasy 96 BioRobot 8000 system (Qiagen). The relative amount of target mRNA was determined by quantitative PCR using the Quantitect SYBR green RT-PCR kit following the manufacturer's instructions (Qiagen) and the following primers: GAPDH forward 5'-GGTATCGTGGAAGGACTCATGAC-3'; GAPDH reverse 5' -ATGCCAGTGAGCTTCCCGTTCAG-3'; ATP6VOD1 forward 5'-TGTCGCAACATCGTGTGGAT-3'; ATP6VDD1 reverse 5'-GAGTGCAATTG AGAGCCTTGG-3'; COPG forward 5'-TCCGCTATGCTGCTGTTCGTA-3'; COPG reverse 5'-GCGGTTTGAATCTGTGACCAG-3'; EIF4A3 forward 5' -TGA TCTTGGCTCCCACAAGAG-3'; EIF4A3 reverse $5^{\prime}$-ATTGGTGCCTCCAATG CAG-3'; NUP98 forward 5' -TTCCGGAATCCGATGTCAGA-3'; NUP98 reverse 5'-TGTAAAGCCTTTGGCCGGACT-3'; NUP205 forward 5'-ACCTTCGGAAG GATCTTCCAA -3'; NUP205 reverse 5'-GGAGTCCCAGAATCACCACAA-3'; NXF1 forward 5' -TGAGCAAACGATACGATGGC-3'; NXF1 reverse 5' -TCTGCG ATTCAGGACAACGTC-3'; SON forward 5'-CAAGCCTTAGAGCTGGCATTG-3'; SON reverse 5'-GCTTGCGTGATTTGTGTTCAG-3'.

The relative expression levels of target mRNA were normalized against control transfected cells. GAPDH was used as an internal standard.

Chemical inhibitors. The chemical inhibitor TG003 (Sigma-Aldrich) directed against the kinase CLK1 was dissolved in DMSO to a concentration of $10 \mathrm{mM}$. Animal experiments. Animals were housed and bred under pathogenfree conditions, BSL 2 according to German Animal Protection Law (Tierschutzgesetz TierSchG). Animal testing was approved by the local authorities (Landesamt für Gesundheit und Soziales Berlin LAGeSo: reference number $\mathrm{G} 0217 / 08)$. C57BL/6/J and $p 27^{-1-}$ mice (B6.129S4-Cdkn1 $\left.1 b^{\text {tmlMlf }} / \mathrm{J}\right)$ were provided by Charles River or bred in house, respectively. Mice aged between 7 and 15 weeks were intranasally infected with influenza A/Puerto Rico/8/34 virus $\left(10 \times \mathrm{LD}_{50}\right.$ (dose lethal to $50 \%$ of animals tested); in $50 \mu \mathrm{l} \mathrm{PBS}$ ). Two days later, lungs of infected animals were isolated and homogenized, followed by centrifugation at $800 \mathrm{~g}$ for $8 \mathrm{~min}$ at $4{ }^{\circ} \mathrm{C}$. The amount of infectious viruses in the supernatant was quantified using the replication assay (see above). Proteins for use in immunoblotting experiments were obtained by adding TRIZOL Reagent (GIBCO BRL) to the remaining cell pellet, according to the manufacturer's instructions.

Data analysis. For identification of primary hits, three parameters were included: luciferase expression, the percentage of infected cells as determined by immunofluorescence microscopy, and the total number of infected cells. The latter parameter was informative because the number of viruses per well correlated with the number of infected cells, with minor influence of cells present. To maximize the robustness of the hit selection and to minimize false positives owing to off-target effects, raw screening data from all three parameters were subjected separately to an analysis pipeline incorporating statistical checkpoints at each step (Supplementary Fig. 4). First, we excluded non-expressed genes by determining constitutive or inducible expression via microarray profiling of non-infected and infected A549 samples (5,814 genes were not expressed). Second, we excluded toxic siRNAs which reduced total cell numbers $(<750$ cells per well) upon transfection (1,520 siRNAs), using the microscopic assay applied throughout the primary screen. Third, non-toxic siRNAs targeting expressed genes were further analysed. For statistical analysis of luciferase assay data obtained from the genome-wide screen, the following plate-wise quality control criteria were used: (1) the average signal from the non-targeting control wells (Allstars) was greater than 10,000 counts; (2) the difference in signal strength between the non-targeting control (Allstars); and (3) the inhibitory control (NP) was at least two orders of magnitude. Using Genedata's Screener software (http:// www.genedata.com), we excluded wells with phenotypes attributable to positional effects. The revised raw data were subjected to statistical analysis using cellHTS ${ }^{31}$, an R-implemented software package for the analysis of cell-based high-throughput RNAi screen data. Raw data were normalized using the B-score method to further exclude positional effects ${ }^{32}$. Next, a $Z$-score transformation was applied to centre and scale the plate-wise data. The medians of the centred and scaled values of at least three independent replicates were used for redundant siRNA activity (RSA) analysis ${ }^{33}$, which applies a rank-based hypergeometric distribution test to identify hits. Only genes for which two corresponding siRNAs were scored as hits were analysed further. Next, Genedata's Screener package was used to select all genes with a robust $Z$-score of less than -2 .

For analysis of hit validation data, the normalized per cent inhibition of infectious virus particles was calculated for each siRNA. Briefly, the difference of each sample value subtracted from the median of the non-targeting control (Allstars) values of the particular plate was divided by the difference of the medians of the non-targeting control and the inhibitory control (siNP). An $80 \%$ normalized inhibition threshold was applied. Genes were scored as validated hits if at least two siRNAs, which did not impair cell viability, fulfilled this criterion.

The ratios of cytosolic to nuclear NP at $5 \mathrm{~h}$ after infection and levels of total NP at $3 \mathrm{~h}$ after infection in samples tested were non-normally distributed. Therefore, to assess the significance of differences between distributions of the target knockdown samples and non-targeting control reference samples (Allstars), we applied the minimal distance estimation Kolmogorov-Smirnov test using the statistical software environment $\mathrm{R}$ (http://www.r-project.org/). The samples sizes are individually defined as the number of main objects per well detected by the automated image analysis package ScanR.

Significant differences in the amount of infectious viruses gained from the lungs of $p 27^{-1-}$ and control mice were tested using a one-tailed $t$-test assuming different standard deviations for the samples and the controls (Welch test).

26. Salomon, R. et al. The polymerase complex genes contribute to the high virulence of the human H5N1 influenza virus isolate A/Vietnam/1203/04. J. Exp. Med. 203, 689-697 (2006).

27. Opitz, B. et al. IFN $\beta$ induction by influenza $A$ virus is mediated by RIG-I which is regulated by the viral NS1 protein. Cell. Microbiol. 9, 930-938 (2007).

28. Falcon, S. \& Gentleman, R. Using GOstats to test gene lists for GO term association. Bioinformatics 23, 257-258 (2007).

29. Cheung, T. K. et al. Generation of recombinant influenza A virus without M2 ionchannel protein by introduction of a point mutation at the 5 ' end of the viral intron. J. Gen. Virol. 86, 1447-1454 (2005).

30. Machuy, N. et al. A global approach combining proteome analysis and phenotypic screening with RNA interference yields novel apoptosis regulators. Mol. Cell. Proteomics 4, 44-55 (2005).

31. Boutros, M., Bras, L. P. \& Huber, W. Analysis of cell-based RNAi screens. Genome Biol. 7, R66 (2006)

32. Brideau, C., Gunter, B., Pikounis, B. \& Liaw, A. Improved statistical methods for hit selection in high-throughput screening. J. Biomol. Screen. 8, 634-647 (2003).

33. König, R. et al. A probability-based approach for the analysis of large-scale RNAi screens. Nature Methods 4, 847-849 (2007). 